\title{
On the Stability of Parabolic Difference Schemes
}

\author{
By Olof B. Widlund
}

1. Introduction. Consider a differential operator of order $2 m$

$$
P(x, \partial)=\sum_{|\nu| \leqq 2 m} A_{\nu}(x) \partial_{1}{ }^{\nu_{1}} \cdots \partial_{s}{ }^{\nu_{s}},
$$

where $\nu=\left(\nu_{1}, \cdots, \nu_{s}\right),|\nu|=\sum \nu_{i}$ and $\partial_{\jmath}=\partial / \partial x_{j} . A_{\nu}(x)$ are complex valued, uniformly bounded $n \times n$ matrices depending on $x=\left(x_{1}, \cdots, x_{s}\right),-\infty<$ $x_{i}<\infty$, in a real vector space $R_{s} . I \cdot \partial / \partial t-P(x, \partial)$, where $I$ is the identity matrix, is said to be uniformly parabolic if there exists a constant $\delta>0$ such that all roots $\sigma_{\mu}$ of

$$
\operatorname{det}\left\{\sum_{|\nu|=2 m} A_{\nu}(x)\left(i \omega_{1}\right)^{\nu_{1}} \cdots\left(i \omega_{s}\right)^{\nu_{s}}-\sigma I\right\}=0
$$

satisfy

$$
\operatorname{Re} \sigma_{\mu} \leqq-\delta
$$

for all $x \in R_{s}$ and all $|\omega|=1{ }^{1}$ Here $\omega \in R_{s}$ is a dual variable.

In this paper we shall study the stability of difference approximations to the initial value problem:

$$
\begin{aligned}
\partial u / \partial t & =P(x, \partial) u, \quad 0 \leqq t \leqq T<\infty, \\
u(x, 0) & =f(x),
\end{aligned}
$$

where $u(x, t)=\left(u_{1}(x, t), \cdots, u_{n}(x, t)\right)^{\prime}$ is a sufficiently smooth vector which for each fixed value of $t$ belongs to a complex Hilbert space $S_{0}$ with an inner product and a norm defined by

$$
\begin{aligned}
(u, v)_{0} & =\int_{R_{s}} u^{*} \cdot v d x, \\
\|u\|_{0}^{2} & =(u, u)_{0} .
\end{aligned}
$$

In the following we shall always suppose that (1.3) is a properly posed initial value problem in the sense of Richtmyer [17], ${ }^{2}$ and only engage in the study of stability conditions for difference approximations. The existence, uniqueness, etc., of solutions of parabolic systems of differential equations have been studied by, among others, Aronson [1], [2], Eìdel'man [4], [5], [6] and Mizohata [15], [16]. We refer the reader to these papers for such questions.

Received July 6, 1964.

${ }^{1} \mathrm{By} y^{\prime}$ and $y^{*}$ we denote the transpose and the conjugate transpose of a vector $y$. Similar notations hold for a matrix $A$. By $|y|$ and $|A|=\sup _{|y|=1}|A y|$ we denote the euclidean norm of $y$ and $A$.

${ }^{2}$ For the basic theory on difference approximations to initial value problems we refer the reader to this book. 
The difference schemes to be studied are of the multistep type:

$$
\begin{array}{r}
u(x,(n+1) k, h)=\sum_{\mu=0}^{r} \alpha_{\mu} u(x,(n-\mu) k, h)+\sum_{\mu=-1}^{r} Q_{\mu}(x, D, h) u(x,(n-\mu) k, h), \\
r \leqq n \leqq T / k-1 ; T / k=\text { integer. }
\end{array}
$$

We suppose that $u(x, \nu k, h)$ are given for $\nu=0,1, \cdots, r-1$, and that $u(x, 0, h)$ $=f(x)$ where $f(x)$ is defined in (1.3). $\alpha_{\mu}$ are real constants. $k$ is the time step which is connected with the space width $h$ by an equation $k=\lambda h^{2 m}, \lambda=$ constant $>0$. $Q_{\mu}(x, D, h)$ are polynomials in the variables $h, h D_{0 j}(h)$ and $h D_{ \pm j}(h)$ with uniformly bounded matrix coefficients. The terms of these polynomials can be written $B_{\mu}{ }^{\sigma \tau}(x) h^{\sigma}(h D)^{\tau}$ with $\sigma+\tau \geqq 2 m$. Here $(h D)^{\tau}=(h D) \cdots(h D)$, where each $h D$ stands for one of the operators $h D_{0 j}(h), h D_{+j}(h), h D_{-j}(h)$. These difference operators are defined by

$$
\begin{aligned}
2 h D_{0 j}(h) u(x) & =u\left(x+h e_{j}\right)-u\left(x-h e_{j}\right), \\
h D_{ \pm j}(h) u(x) & = \pm\left(u\left(x \pm h e_{j}\right)-u(x)\right),
\end{aligned}
$$

where $e_{j}$ is the unit vector in the direction of the $j$ th coordinate axis. The elements of $B_{\mu}{ }^{\sigma \tau}(x)$ are supposed to have sufficiently many uniformly bounded derivatives. The sum of the terms for which $\sigma=0$ and $\tau=2 m$ are denoted $Q_{\mu}{ }^{(1)}(x, D, h)$, respectively.

Following Richtmyer [17] we introduce a new inner product space $S_{r}$. The elements of $S_{r}$ are $(r+1)$-component vectors, whose components are elements in $S_{0}$. The inner product and the norm are defined by

$$
\begin{aligned}
(\tilde{u}, \tilde{v})_{r} & =\sum_{i=0}^{r}\left(u_{i}, v_{i}\right)_{0}, \\
\|\tilde{u}\|_{r}^{2} & =(\tilde{u}, \tilde{u})_{r}, \\
\tilde{u} & =\left(u_{0}, u_{1}, \cdots, u_{r}\right)^{\prime}, \\
\tilde{v} & =\left(v_{0}, v_{1}, \cdots, v_{r}\right)^{\prime}, \quad u_{i} \in S_{0}, v_{i} \in S_{0}, i=0,1, \cdots, r .
\end{aligned}
$$

We shall always suppose that $\left(I-Q_{-1}(x, D, h)\right)^{-1}$ and $\left(I-Q_{-1}\left(x_{0}, D, h\right)\right)^{-1}$, $x_{0} \in R_{s}$, exist and that they are defined for all $u \in S_{0}$. We also suppose that these operators are uniformly bounded, that is, the bound for $\left\|\left(I-Q_{-1}(x, D, h)\right)^{-1}\right\|_{0}$ is independent of $h$ and the bound for $\left\|\left(I-Q_{-1}\left(x_{0}, D, h\right)\right)^{-1}\right\|_{0}$ is independent of $x_{0}$ and $h$. We are then able to transform (1.4) into an explicit one-step formula:

$$
\begin{aligned}
& \tilde{u}(x,(n+1) k, h)=\tilde{Q} \tilde{u}(x, n k, h), \\
& \tilde{Q}=\left(\begin{array}{ccc}
\left(I-Q_{-1}\right)^{-1}\left(\alpha_{0} I+Q_{0}\right) & \ldots \ldots \ldots & \left(I-Q_{-1}\right)^{-1}\left(\alpha_{r} I+Q_{r}\right) \\
I & \ldots \ldots \cdots & 0 \\
0 & 0 \ldots \ldots \ldots & 0
\end{array}\right) \\
& \tilde{u}(x, n k, h)=(u(x, n k, h), \cdots, u(x,(n-r) k, h))^{\prime} .
\end{aligned}
$$

As in the case of differential equations many properties of difference schemes are stated in terms of characteristic matrices. We therefore, denoting by 


$$
\xi_{j}=\omega_{j} h, \quad j=1, \cdots, s
$$

define the matrices $\hat{Q}_{\mu}(x, \xi, h), \hat{Q}_{\mu}{ }^{(1)}(x, \xi, h)$ and $\hat{Q}(x, \xi, h)$ which are constructed by exchanging $h D_{0 j}(h)$ and $h D_{ \pm j}(h)$ for $i \cdot \sin \xi_{j}$ and $2 i \cdot \sin (1 / 2) \xi_{j} \cdot e^{ \pm i(1 / 2) \xi_{j}}$, respectively, in $Q_{\mu}(x, D, h), Q_{\mu}{ }^{(1)}(x, D, h)$ and $\widetilde{Q}(x, D, h)$. In the case when $Q_{\mu}$ do not depend on $x$ this amounts to the same thing as taking the Fourier transforms for

$$
\begin{gathered}
\left(h D_{0 j}(h) f(x)\right)^{\wedge}=i \cdot \sin \xi_{j} \cdot \hat{f}, \\
\left(h D_{ \pm j}(h) f(x)\right)^{\wedge}=2 i \cdot \sin (1 / 2) \xi_{j} \cdot e^{ \pm(1 / 2) \xi_{j}} \cdot \hat{f}, \\
\hat{f}=\text { Fourier transform of } f(x) .
\end{gathered}
$$

It follows immediately that $\hat{Q}_{\mu}, \hat{Q}_{\mu}^{(1)}$ and $\hat{Q}$ are $2 \pi$-periodic matrix-valued functions of $\xi_{j}, j=1, \cdots, s$. Observe especially that $\hat{Q}_{\mu}{ }^{(1)}$ depend only on $\xi$ and $x$ and that $\hat{O}_{\mu}^{(1)}=O\left(|\xi|^{2 m}\right)$.

We write $\hat{Q}$ as the sum of three matrices:

where

$$
\hat{Q}=\hat{Q}^{(0)}+\hat{Q}^{(1)}+\hat{Q}^{(2)}
$$

$$
\hat{Q}^{(0)}=\left(\begin{array}{ccccc}
\alpha_{0} I & \alpha_{1} I & \cdots & \alpha_{r-1} I & \alpha_{r} I \\
I & 0 & \cdots & 0 & 0 \\
& \cdots \cdots \cdots & \cdots & \\
0 & 0 & \cdots & I & 0
\end{array}\right)
$$

and

$$
\hat{Q}^{(0)}+\hat{Q}^{(1)}=\left(\begin{array}{ccc}
\left(I-\hat{Q}_{-1}^{(1)}\right)^{-1}\left(\alpha_{0} I+\hat{Q}_{0}^{(1)}\right) & \cdots & \left(I-\hat{Q}_{-1}^{(1)}\right)^{-1}\left(\alpha_{r} I+\hat{Q}_{r}{ }^{(1)}\right) \\
I & \ldots \ldots & 0 \\
& \cdots \cdots & \\
0 & \cdots \cdots I & 0
\end{array}\right) .
$$

Observe that the existence of $\left(I-\hat{Q}_{-1}^{(1)}\right)^{-1}$ follows from the existence of $\left(I-\hat{Q}_{-1}\right)^{-1}$, the fact that $\hat{Q}_{-1}^{(1)}$ depends on $\xi$ and $x$ only and the fact that $\hat{Q}_{-1}-\hat{Q}_{-1}^{(1)}=O(h)$. We also introduce the $(r+1) \times(r+1)$ matrix

$$
Q=\left(\begin{array}{ccccc}
\alpha_{0} & \alpha_{1} & \cdots & \alpha_{r} \\
1 & 0 & \cdots & 0 & 0 \\
& \cdots \cdots & & \\
0 & 0 & \cdots & 1 & 0
\end{array}\right)
$$

The stability conditions of this paper are stated in terms of $\kappa_{i},\left|\kappa_{j}\right| \leqq$ $\left|\kappa_{i}\right|, 1 \leqq i<j \leqq(r+1) n$, the eigenvalues of $\hat{Q}^{(0)}+\hat{Q}^{(1)}$ and $\gamma_{i},\left|\gamma_{j}\right| \leqq\left|\gamma_{i}\right|$, $1 \leqq i<j \leqq r+1$, the eigenvalues of $Q$. This is very convenient from a practical point of view. Observe that $\kappa_{i}$ can be calculated as the roots of

$$
\operatorname{det}\left\{\kappa^{r+1}\left(I-\hat{Q}_{-1}^{(1)}\right)-\kappa^{r}\left(\alpha_{0} I+\hat{Q}_{0}^{(1)}\right)-\cdots-\left(\alpha_{r} I+\hat{Q}_{r}^{(1)}\right)\right\}=0 .
$$

Following Richtmyer [17] we define:

Definition 1. (1.4) is a consistent difference approximation to (1.3) if for all sufficiently smooth solutions $u(x, t)$ to $(I \cdot \partial / \partial t-P(x, \partial)) u=0$ :

$$
\left\|\left(I-Q_{-1}\right) u(x, t+k)-\sum_{\mu=0}^{r}\left(\alpha_{\mu} I+Q_{\mu}\right) u(x, t-\mu k)\right\|_{0}=o(k) .
$$


Using Taylor's theorem we immediately obtain that if (1.4) is a consistent difference approximation:

$$
\begin{aligned}
\sum_{\mu=0}^{r} \alpha_{\mu} & =1 \\
\left(1+\sum_{\mu=0}^{r} \mu \alpha_{\mu}\right) k P(x, \partial) & =\sum_{\mu=-1}^{r} Q_{\mu}+o(k) .
\end{aligned}
$$

Because of the fact that various stability definitions are used in the literature, we here give two definitions.

Definition 2. (1.6) is a stable difference scheme if there exists a constant $C_{1}$ independent of $h$ such that for all $\tilde{u}_{r} \in S_{r}$

$$
\left\|\tilde{u}_{n}\right\|_{r} \leqq C_{1}\left\|\tilde{u}_{r}\right\|_{r}
$$

for all $n \leqq T / k, \tilde{u}_{m}=(u(x, m k, h), \cdots, u(x,(m-r) k, h))^{\prime}$.

Definition 3. (1.6) is a strongly stable difference scheme if there exists a constant $C_{2}$, independent of $h$, and a norm $\|\cdot\|_{\text {eq }}$, equivalent to $\|\cdot\|_{r}$, such that

$$
\left\|\tilde{u}_{n+1}\right\|_{\mathrm{eq}} \leqq\left(1+C_{2} k\right)\left\|\tilde{u}_{n}\right\|_{\mathrm{eq}}
$$

for all $n<T / k$.

(1.12) is easily seen to be a condition of at least the same strength as (1.11). The significance of strong stability is thoroughly discussed in a paper by Kreiss [12].

In analogy to (1.1) and (1.2) we define:

Definition 4. (1.6) is a parabolic difference scheme if there exists a constant $\delta_{1}>0$ such that for all $\left|\xi_{j}\right| \leqq \pi$ and all $x \in R_{s}$

$$
\left|\kappa_{i}\right| \leqq 1-\delta_{1}|\xi|^{2 m}, \quad i=1, \cdots,(r+1) n .
$$

Remark. If we choose $\alpha_{0}=1, \alpha_{1}=\alpha_{2}=\cdots=\alpha_{r}=0$ and

$$
\begin{aligned}
& Q_{0}=\lambda \sum_{|\nu| \leqq 2 m} A_{\nu}(x) h^{2 m-|\nu|}\left(h D_{01}(h)\right)^{\nu_{1}} \cdots\left(h D_{0 s}(h)\right)^{\nu s}, \\
& Q_{\mu}=0, \quad \mu \geqq 1, \quad Q_{-1}=0,
\end{aligned}
$$

we get, as is easily verified using (1.2), a consistent parabolic difference approximation to (1.3) provided $\lambda$ is chosen sufficiently small. This shows that there always exists a consistent parabolic difference scheme to each uniformly parabolic differential operator.

We are now able to state our results.

Theorem 1. Let (1.4) be a consistent difference approximation to (1.3). Under the assumptions

(a) $I \cdot \partial / \partial t-P(x, \partial)$ is uniformly parabolic,

(b) the eigenvalues $\gamma_{i}, i=2, \cdots, r+1$, lie in the interior of the unit circle,

(c) for every $\xi \neq 0$, all eigenvalues $\kappa_{i}$ of $\hat{Q}^{(0)}+\hat{Q}^{(1)}$ lie in the interior of the unit circle,

the difference scheme is parabolic.

Theorem 2. Suppose that (1.6) is a parabolic difference scheme and that all eigenvalues $\gamma_{i}$ of $a$ with modulus $=1$ are simple. Then it is strongly stable. 
Observe that in Theorem 2 we are able to weaken the conditions on $\gamma_{i}$ compared with those in Theorem 1.

In Section 2 we prove Theorem 1. In Section 3 we prove, using results by Kreiss [12], [13], a fundamental inequality, which essentially corresponds to Theorem 2 in the case when $\widetilde{Q}$ has constant coefficients. In Section 4 we construct a new norm and state some lemmas. Using the results of Section 3 and Section 4 we are able to give a proof of Theorem 2 in Section 5. Here we make use of a well-known device due to Gårding [7].

For convenience we only treat homogeneous equations with coefficients independent of $t$. The extension of the results of this paper to the general case, however, presents no new difficulties.

The techniques used in this paper are closely related to those used by Lax and Wendroff [14] and Kreiss [10]-[13]. A slightly different approach to difference approximations to parabolic differential equations is found in papers by John [9] and Aronson [3]. Using a similar type of condition as we do, John [9], working with a maximum norm, proved the stability of difference approximations to a single second order parabolic differential equation.

I am very grateful to Heinz-Otto Kreiss who proposed this problem to me and who guided my work. He also gave me the opportunity to read the manuscript of the paper by P. D. Lax and B. Wendroff mentioned above, which the authors had kindly put at his disposal. I also want to thank Lars Hörmander for a clarifying discussion on Gårding's [7] device.

2. Proof of Theorem 1. First observe that because of (c) we only have to consider a small neighborhood of $\xi=0$. Using (b) and (1.9) we see that $\gamma_{1}=1$. Since the eigenvalues depend continuously on the matrix, (b) also tells us that we only have to study the eigenvalues $\kappa_{i}(\xi)$ close to $\gamma_{1}=1$. Put $\eta=\kappa-1$. Using (1.9) we get from (1.8):

$$
\operatorname{det}\left\{\eta\left(1+\sum_{\mu=0}^{r} \alpha_{\mu} \mu\right)-\sum_{\mu=-1}^{r} \hat{Q}_{\mu}^{(1)}+O\left(\eta|\xi|^{2 m}\right)+O\left(\eta^{2}\right)\right\}=0 .
$$

(1.4) is a consistent approximation to (1.3). This easily establishes

$$
\left(1+\sum_{\mu=0}^{r} \mu \alpha_{\mu}\right) \lambda \sum_{|\nu|=2 m} A_{\nu}(x)\left(i \xi_{1}\right)^{\nu_{1}} \cdots\left(i \xi_{s}\right)^{\nu_{s}}=\sum_{\mu=-1}^{r} \hat{Q}_{\mu}^{(1)}+o\left(|\xi|^{2 m}\right)
$$

Substitute (2.2) into (2.1). Using (1.1) and (1.2) we are able to prove (1.13) at once.

3. A Fundamental Inequality. The purpose of this section is to establish the following lemma.

Lemma 1. Suppose that the assumptions of Theorem 2 hold. Then, for each $x_{0} \in R_{s}$, there exists a norm $\|\cdot\|_{H\left(x_{0}\right)}$ equivalent to $\|\cdot\|_{r}$ such that, for all $u \in S_{r}$ :

$$
\|u\|_{H\left(x_{0}\right)}^{2}-\left\|\left(\widetilde{Q}^{(0)}+\widetilde{Q}^{(1)}\left(x_{0}, D, h\right)\right) u\right\|_{H\left(x_{0}\right)}^{2} \geqq \delta\left(L_{m, H\left(x_{0}\right)}(u)\right)^{2},
$$

where $\delta>0$ is a constant independent of $x_{0}$, and

$$
\left(L_{q, H\left(x_{0}\right)}(u)\right)^{2}=h^{2 q} \sum_{j=1}^{s}\left\|D_{+j}^{q} u\right\|_{H\left(x_{0}\right)}^{2}
$$


and such that there is a constant $C_{3}>0$, independent of $x_{0}$, for which

$$
C_{3}^{-1}\|u\|_{r}^{2} \leqq\|u\|_{H\left(x_{0}\right)}^{2} \leqq C_{3}\|u\|_{r}^{2}
$$

The proof of Lemma 1 is based on Lemma 2 given by Kreiss [12].

Lemma 2 (Kreiss). Let $f$ denote a family of $m^{\prime} \times m^{\prime}$ matrices. Then the following statements are equivalent:

(1) There is a constant $C_{1}$ such that for all $A \in f$ :

$$
\left|A^{\nu}\right| \leqq C_{1}, \quad \nu=1,2, \cdots \text {. }
$$

(2) There is a constant $C_{2}$, such that for all complex $s,|s|<1,(s A-I)^{-1}$ exists and

$$
\left|(s A-I)^{-1}\right| \leqq C_{2} /(1-|s|) \text {. }
$$

(3) There is a constant $C_{3}>0$ and to every $A \in f$ a positive definite hermitian matrix $\hat{H}$ with

$$
C_{3}^{-1} I \leqq \hat{H} \leqq C_{3} I^{3}
$$

such that

$$
\begin{aligned}
A^{*} \hat{H} A & \leqq(1-\rho) \hat{H}, \quad 2 \rho=\min _{j}\left(1-\left|\kappa_{j}\right|\right), \\
\kappa_{j} & =\text { the } j \text { th eigenvalues of } A .
\end{aligned}
$$

Here $\hat{H}=U^{*} S^{*} D S U$, where $U$ is any unitary matrix which transforms $A$ into upper diagonal form, $S$ is a matrix the elements of which are rational functions of the elements of $U^{*} A U$ and $U^{*} A^{*} U$ and $D$ is a diagonal matrix independent of $A$.

Lemma 2 is a slight modification of the original lemma due to Kreiss. In [12] he only proved that $A^{*} \hat{H} A \leqq \hat{H}$. The modification of the proof is, however, a trivial one.

To prove Lemma 1 we first show that the second statement in Lemma 2 holds for $\hat{Q}^{(0)}+\hat{Q}^{(1)}\left(x_{0}, \xi\right)$. As before we only have to consider a small neighborhood of $\xi=0$. The assumptions on the eigenvalues $\gamma_{i}$ of $Q$ imply that there is, for every $\epsilon>0$, a nonsingular matrix $S$ such that

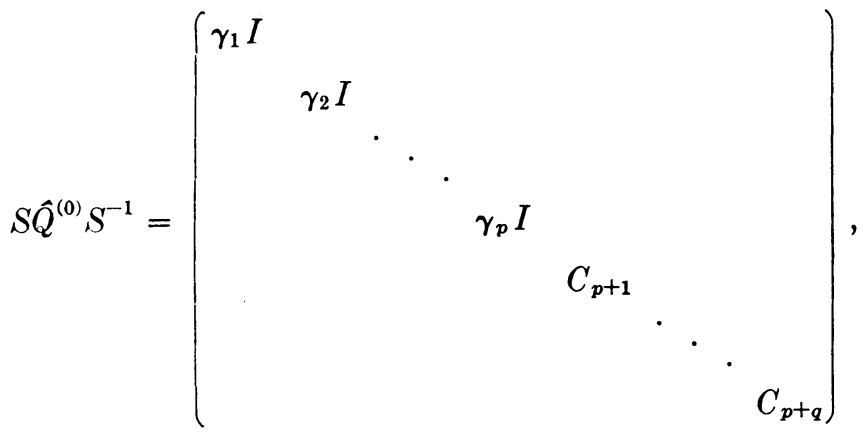

${ }^{3} A \leqq B$ means that $y^{*} A y \leqq y^{*} B y$ for all vectors $y$. 
where

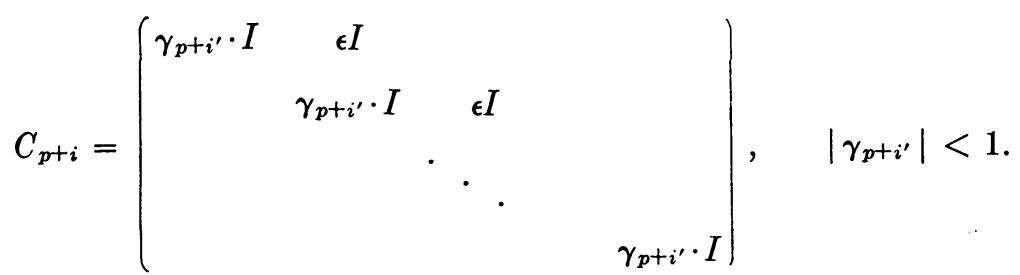

Taking into account that $\hat{Q}^{(1)}=O\left(|\xi|^{2 m}\right)$ it follows that

$$
S\left(\hat{Q}^{(0)}+\hat{Q}^{(1)}\right) S^{-1}=S \hat{Q}^{(0)} S^{-1}+O\left(|\xi|^{2 m}\right) .
$$

Therefore by Gershgorin's estimate of the eigenvalues of a matrix we have

$$
\begin{aligned}
\left|\kappa_{i}-\gamma_{i^{\prime}}\right| \leqq \text { const. }|\xi|^{2 m} & \text { if } \quad\left|\gamma_{i^{\prime}}\right|=1, \quad i=i^{\prime} \cdot n-i^{\prime \prime}, 0 \leqq i^{\prime \prime}<n . \\
\left|\kappa_{i}-\gamma_{i^{\prime}}\right| \leqq \text { const. }|\xi|^{2 m}+\epsilon & \text { if }\left|\gamma_{i^{\prime}}\right|<1,
\end{aligned}
$$

We can now write

$s S\left(\hat{Q}^{(0)}+\hat{Q}^{(1)}\right) S^{-1}-I$

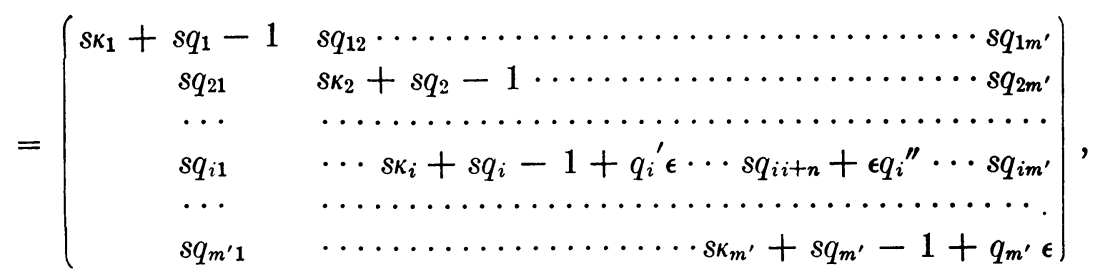

where $m^{\prime}=n(r+1), q_{i}{ }^{\prime}=q_{i}{ }^{\prime \prime}=0$ for $i \leqq p n$ and $\left|q_{i}{ }^{\prime}\right| \leqq 1, q_{i}{ }^{\prime \prime}=0$ or 1 for $i>p n$. At this

$$
\left|q_{i}\right|+\left|q_{i k}\right| \leqq \text { const. }|\xi|^{2 m}
$$

where because of the uniform bound for the coefficients of $\hat{Q}^{(1)}$, the constant can be chosen independent of $x_{0}$. Denote by $c_{k l}$ the elements of $\left(s S\left(\hat{Q}^{(0)}+\hat{Q}^{(1)}\right) S^{-1}-I\right)^{-1}$ and by $D_{k l}$ the matrix obtained from $s S\left(\hat{Q}^{(0)}+\hat{Q}^{(1)}\right) S^{-1}-I$ by omitting the $k$ th row and the $l$ th column. Then by Cramer's rule:

$$
c_{k l}=(-1)^{k+l} \operatorname{det} D_{l k} / \operatorname{det}\left(s S\left(\hat{Q}^{(0)}+\hat{Q}^{(1)}\right) S^{-1}-I\right) .
$$

Observe that

$$
\operatorname{det}\left(s S\left(\hat{Q}^{(0)}+\hat{Q}^{(1)}\right) S^{-1}-I\right)=\prod_{i=1}^{m^{\prime}}\left(s \kappa_{i}-1\right) .
$$

From (1.13) and the assumptions of Theorem 2 follows:

$$
\begin{aligned}
& \left|s \kappa_{i}-1\right| \geqq 1-\left|s \kappa_{i}\right| \geqq \delta_{1}|\xi|^{2 m}, \quad i \leqq p n,|s|<1, \\
& \left|s \kappa_{i}-1\right| \geqq \text { const., } \quad i>p n,|s|<1,
\end{aligned}
$$

for sufficiently small $\epsilon>0$. We also know that

$$
\left|s \kappa_{i}-1\right| \geqq 1-|s| .
$$


Using elementary properties of a determinant we obtain from (3.9)

$$
c_{k l}=(-1)^{k+l} \operatorname{det} E_{l k} /\left(s \kappa_{l}-1\right),
$$

where we construct $E_{l k}$ by dividing the $i$ th row of $s S\left(\hat{Q}^{(0)}+\hat{Q}^{(1)}\right) S^{-1}-I$ by $s \kappa_{i}-1$, respectively, and finally omitting the $l$ th row and the $k$ th column. Now use (3.8), (3.10) and (3.11). This easily shows that

$$
\left|c_{k l}\right| \leqq \text { const. } /(1-|s|)
$$

with the constant chosen independent of $x_{0}$ and $\xi$. Using Lemma 2 we obtain

$$
\left|\left(\hat{Q}^{(0)}+\hat{Q}^{(1)}\left(x_{0}, \xi\right)\right)^{\nu}\right| \leqq C_{1}, \quad \nu=1,2, \cdots,
$$

or from (3.6), (3.7) and (1.13):

$$
\begin{aligned}
\left(\hat{Q}^{(0)}+\hat{Q}^{(1)}\left(x_{0}, \xi\right)\right)^{*} \hat{H}\left(x_{0}, \xi\right)\left(\hat{Q}^{(0)}\right. & \left.+\hat{Q}^{(1)}\left(x_{0}, \xi\right)\right) \\
& \leqq \hat{H}\left(x_{0}, \xi\right)\left(1-\delta_{1} / 2|\xi|^{2 m}\right)
\end{aligned}
$$

and

$$
C_{3}^{-1} I \leqq \hat{H}\left(x_{0}, \xi\right) \leqq C_{3} I,
$$

where $C_{3}>0$ is a constant independent of $x_{0}$ and $\hat{H}\left(x_{0}, \xi\right)$ is a positive definite hermitian matrix. Because of the fact that the matrix $U$ in Lemma 2 can be chosen as a piecewise continuous matrix-valued function of the elements of $\hat{Q}^{(0)}+\hat{Q}^{(1)}$ it follows from Lemma 2 that we are able to construct an operator $H\left(x_{0}\right)$ by

$$
H\left(x_{0}\right) u(x)=(2 \pi)^{-s / 2} \int_{R_{\theta}} \hat{H}\left(x_{0}, \xi\right) e^{i \omega^{\prime} x} \hat{u}(\omega) d \omega,
$$

where

$$
\hat{u}(\omega)=(2 \pi)^{-s / 2} \int_{R_{s}} e^{-i \omega^{\prime} x} u(x) d x .
$$

We now define the new norm by

$$
\|u\|_{H\left(x_{0}\right)}^{2}=\left(u, H\left(x_{0}\right) u\right)_{r} .
$$

Using Parseval's relation and (3.13) we obtain (3.3). Observe that

$$
\left(L_{q, H\left(x_{0}\right)}(u)\right)^{2}=\int_{R_{s}} \sum_{j}\left(2 \sin \xi_{j} / 2\right)^{2 q} \hat{u}^{*}(\omega) \hat{H}\left(x_{0}, \xi\right) \hat{u}(\omega) d \omega,
$$

and that

$$
\left|\xi_{j}\right| \geqq\left|2 \sin \xi_{j} / 2\right| \text {. }
$$

(3.1) is now established using Parseval's relation, (3.12) and (3.17).

4. Some Lemmas. Here we collect some lemmas, which we need in Section 5 .

Lemma 3. There exists a function $\phi(x) \in C^{\infty}\left(R_{s}\right)$ such that, ${ }^{4}$ the support of $\phi(x)$. is contained in the cube defined by $\max _{\nu}\left|x_{v}\right|<1, \phi(x) \geqq 0$ and

$$
1=\sum_{0} \phi^{2}(x-g),
$$

${ }^{4} \mathrm{By} C^{\infty}\left(R_{s}\right)$ we denote the set of all functions defined in $R_{s}$, whose partial derivatives of all orders exist. 
where $g$ runs through all points $g=\left(g_{1}, \cdots, g_{s}\right)$ with integral coordinates.

Proof. Hörmander [8] showed that there exists a non-negative function $\theta(x) \in$ $C^{\infty}\left(R_{s}\right)$ with support contained in the cube $\max _{\nu}\left|x_{\nu}\right|<1$ such that $\theta(x) \geqq 0$ and

$$
1=\sum_{g} \theta(x-g)
$$

Then

$$
\phi(x)=\theta(x) / \sqrt{\sum_{g} \theta^{2}(x-g)}
$$

is easily seen to have the required properties.

For a given $\epsilon>0$ and any $u(x) \in S_{r}$ now define:

$$
u_{g}(x)=\phi((x-\epsilon g) / \epsilon) u(x) .
$$

Observe that at most $2^{s}$ vector-valued functions $u_{g}$ can be different from 0 at any point $x \in R_{s}$.

Lemma 4. Let

$$
(u, H v)=\sum_{g}\left(u_{g}, H\left(x_{g}\right) v_{g}\right)_{r}, \quad u, v \in S_{r}, x_{g}=\epsilon g,
$$

where $u_{g}$ and $v_{g}$ are defined by (4.2) and $H\left(x_{g}\right) v_{g}$ is defined by (3.14). Then $(u, H v)$ is an inner product defining an equivalent norm which satisfies

$$
C_{3}^{-1}\|u\|_{r}^{2} \leqq(u, H u) \leqq C_{3}\|u\|_{r}^{2}
$$

where $C_{3}$ is the constant in (3.3).

Proof. $(u, H v)$ is easily seen to be an inner product. (4.4) follows from (3.3) and (4.1).

Lemma 5. Let $p$ and $q, p \geqq q$, be positive integers. Then

$$
L_{p, H\left(x_{g}\right)}(u) \leqq 2^{p-q} L_{q, H\left(x_{g}\right)}(u) .
$$

Proof. The inequality follows from:

$$
\begin{aligned}
\left(\left(h D_{+j}(h)\right)^{p} u, H\left(x_{g}\right)\left(h D_{+j}(h)\right)^{p} u\right)_{r} & =\int_{R_{g}} \hat{u}^{*} \hat{H}\left(x_{g}\right) \hat{u}\left(2 \sin \xi_{j} / 2\right)^{2 p} d \omega \\
& \leqq \int_{R_{g}} 4^{p-q} \hat{u}^{*} \hat{H}\left(x_{g}\right) \hat{u}\left(2 \sin \xi_{j} / 2\right)^{2 q} d \omega \\
& =4^{p-q}\left(\left(h D_{+j}(h)\right)^{q} u, H\left(x_{g}\right)\left(h D_{+j}(h)\right)^{q} u\right)_{r} .
\end{aligned}
$$

Lemma 6. For any $\epsilon_{1}>0$ there exists a constant $C\left(\epsilon_{1}\right)$ such that if $p>q$ :

$$
h^{2 p-2 q}\left(L_{q, H\left(x_{o}\right)}(u)\right)^{2} \leqq \epsilon_{1}\left(L_{p, H\left(x_{o}\right)}(u)\right)^{2}+C\left(\epsilon_{1}\right) h^{2 p}\left(L_{0, H\left(x_{o}\right)}(u)\right)^{2} .
$$

Proof. The lemma follows from the following elementary inequality:

$$
h^{2 p-2 q}\left(2 \sin \xi_{j} / 2\right)^{2 q} \leqq \epsilon_{1}\left(2 \sin \xi_{j} / 2\right)^{2 p}+C\left(\epsilon_{1}\right) h^{2 p} .
$$

Observing that the difference operators commute with $H\left(x_{g}\right)$ we establish the following lemma at once. 
LEMMA 7.

$$
\begin{aligned}
\left(u, H\left(x_{g}\right) A\left(h D_{0 j}(h) v\right)\right)_{r}= & -\left(h D_{0 j}(h) u, H\left(x_{g}\right) A v\right)_{r} \\
& -h / 2\left(E_{j}^{-1} u, H\left(x_{g}\right)\left(E_{j}^{-\theta_{1}} \partial A / \partial x_{j}\right) v\right)_{r} \\
& -h / 2\left(E_{j} u, H\left(x_{g}\right)\left(E_{j}^{\theta_{2}} \partial A / \partial x_{j}\right) v\right)_{r}, \\
\left(u, H\left(x_{g}\right) A h D_{+j}(h) v\right)_{r}= & -\left(h D_{-j}(h) u, H\left(x_{g}\right) A v\right)_{r} \\
& -\left(E_{j}^{-1} u, H\left(x_{g}\right)\left(E_{j}^{-\theta_{3}} \partial A / \partial x_{j}\right) v\right)_{r} h,
\end{aligned}
$$

where $0<\theta_{1}, \theta_{2}, \theta_{3}<1, A(x)$ is a sufficiently smooth matrix and $E_{j} u(x)=$ $u\left(x+h e_{j}\right)$.

Lemma 8. Let $(h D)^{p}$ denote the product of $p$ operators $h D$, where each $h D$ stands for one of the operators $h D_{0 j}, h D_{+j}$ or $h D_{-j}$. Then

$$
L_{0, H\left(x_{\boldsymbol{\sigma}}\right)}\left((h D)^{p} u\right) \leqq L_{p, H\left(x_{\boldsymbol{q}}\right)}(u) .
$$

Proof.

$$
\begin{aligned}
\left(L_{0, H\left(x_{g}\right)}\left((h D)^{p} u\right)\right)^{2} & \leqq \int_{R_{g}} \hat{u}^{*} \hat{H}\left(x_{g}\right) \hat{u}\left(\max \left(2 \sin \xi_{j} / 2\right)\right)^{2 p} d \omega \\
& \leqq \sum_{j} \int_{R_{\text {a }}} \hat{u}^{*} \hat{H}\left(x_{g}\right) \hat{u}\left(2 \sin \xi_{j} / 2\right)^{2 p} d \omega=\left(L_{p, H\left(x_{g}\right)}(u)\right)^{2} .
\end{aligned}
$$

Lemma 9. Let $(h D)^{p}$ be defined as in Lemma 8. Denote by $\overline{(h D)}^{p}$ the operator constructed by exchanging $h D_{+j}$ for $h D_{-j}$ and $h D_{-j}$ for $h D_{+j}$ in $(h D)^{p}$. Let $A(x)$ be a sufficiently smooth matrix such that the derivatives of order $\leqq p_{1} \leqq m$ of the elements of $A(x)$ are uniformly bounded. For any $\epsilon_{2}>0$ there exists a constant $C\left(\epsilon_{2}\right)$ independent of $h, \dot{g}, u$ and $v$ such that

$$
\begin{aligned}
& h^{m-p_{1}}\left|\left(u, H\left(x_{g}\right) A(h D)^{p_{1}}(h D)^{m} v\right)_{r}-(-1)^{p_{1}}\left(\overline{(h D)}^{p_{1}} u, H\left(x_{o}\right) A(h D)^{m} v\right)_{r}\right| \\
& \leqq \epsilon_{2}\left(\left(L_{m, H\left(x_{g}\right)}(u)\right)^{2}+\left(L_{m, H\left(x_{g}\right)}(v)\right)^{2}\right)+C\left(\epsilon_{2}\right)\left(\left(L_{0, H\left(x_{g}\right)}(u)\right)^{2}+\left(L_{0, B\left(x_{g}\right)}(v)\right)^{2}\right) h^{2 m} .
\end{aligned}
$$

Proof. Using Lemma 7, Lemma 8 and Schwarz's inequality several times it is possible to show that

$$
\begin{aligned}
\mid\left(u, H\left(x_{g}\right) A(h D)^{p_{1}}(h D)^{m} v\right)_{r} & -(-1)^{p_{1}}\left(\overline{(h D)} \bar{p}^{p_{1}} u, H\left(x_{g}\right) A(h D)^{m} v\right)_{r} \mid \\
& \leqq \text { const. } \sum_{\nu_{2} \leqq m ; \nu_{1}<p_{1}} h^{p_{1}+m-\left(\nu_{1}+\nu_{2}\right)} L_{\nu_{1}, H\left(x_{g}\right)}(u) L_{\nu_{2}, H\left(x_{g}\right)}(v) .
\end{aligned}
$$

We now use the elementary inequality

$$
a b \leqq a^{2} / 2 c+b^{2} c / 2
$$

and Lemma 5 and Lemma 6 to finish the proof.

5. Proof of Theorem 2. The proof is carried out in three steps. At this we use arguments analogous to those of Hörmander [8].

First consider:

$$
\begin{aligned}
& \left(L_{0, H\left(x_{g}\right)}\left(u_{g}\right)\right)^{2}-\left(L_{0, H\left(x_{g}\right)}\left(\widetilde{Q}\left(x_{g}, D, h\right) u_{g}\right)\right)^{2} \\
& =\left(L_{0, H\left(x_{\theta}\right)}\left(u_{g}\right)\right)^{2}-\left(L_{0, H\left(x_{g}\right)}\left(\left(\widetilde{Q}^{(0)}+\widetilde{Q}^{(1)}\left(x_{g}, D, h\right)\right) u_{g}\right)\right)^{2}-I-I I-I I I .
\end{aligned}
$$


Here

$$
\begin{aligned}
\mathrm{I} & =2 \operatorname{Re}\left(\widetilde{Q}^{(0)} u_{g}, H\left(x_{g}\right) \widetilde{Q}^{(2)}\left(x_{q}, D, h\right) u_{g}\right)_{r}, \\
\mathrm{II} & =2 \operatorname{Re}\left(\widetilde{Q}^{(1)}\left(x_{q}, D, h\right) u_{g}, H\left(x_{q}\right) \widetilde{Q}^{(2)}\left(x_{q}, D, h\right) u_{g}\right)_{r}, \\
\mathrm{III} & =\left(L_{0, \boldsymbol{B}\left(x_{\boldsymbol{g}}\right)}\left(\widetilde{Q}^{(2)}\left(x_{g}, D, h\right) u_{g}\right)\right)^{2} .
\end{aligned}
$$

Now observe that from the uniform boundedness of $\left(I-Q_{-1}\left(x_{g}, D, h\right)\right)^{-1}$ follows, $\left\|\left(I_{\dot{j}}-Q_{-1}^{(1)}\left(x_{g}, D, h\right)\right) v\right\|_{0}+\left\|Q_{-1}\left(x_{g}, D, h\right) v-Q_{-1}^{(1)}\left(x_{g}, D, h\right) v\right\|_{0}$

$$
\geqq\left\|\left(I-Q_{-1}\left(x_{g}, D, h\right)\right) v\right\|_{0} \geqq \text { const. }\|v\|_{0} .
$$

Using Lemma 5 we easily show that

$$
\left\|Q_{-1}\left(x_{g}, D, h\right) v-Q_{-1}^{(1)}\left(x_{g}, D, h\right) v\right\|_{0} \leqq h \text { const. }\|v\|_{0} .
$$

This shows that $\left(I-Q_{-1}^{(1)}\left(x_{g}, D, h\right)\right)^{-1}$ is a uniformly bounded operator if $h$ is sufficiently small. Also observe that

$$
\begin{aligned}
& \left(I-Q_{-1}\right)^{-1}\left(\alpha_{\mu} I+Q_{\mu}\right)-\left(I-Q_{-1}^{(1)}\right)^{-1}\left(\alpha_{\mu} I+Q_{\mu}^{(1)}\right) \\
& \quad=\left(I-Q_{-1}\right)^{-1}\left(Q_{-1}-Q_{-1}^{(1)}\right)\left(I \stackrel{-}{-} Q_{-1}^{(1)}\right)^{-1}\left(\alpha_{\mu} I+Q_{\mu}^{(1)}\right)+\left(I-Q_{-1}\right)^{-1}\left(Q_{\mu}-Q_{\mu}^{(1)}\right) .
\end{aligned}
$$

Here

$$
Q_{\mu}-Q_{\mu}^{(1)}=\sum_{\sigma>0 ; \tau+\sigma \geq 2 m} B_{\mu}{ }^{\sigma \tau} h^{\sigma}(h D)^{\tau} .
$$

Using Schwarz's inequality, Lemmas $5,6,8$ and 9 we easily show that,

$$
|\mathrm{I}|+|\mathrm{II}|+|\mathrm{III}| \leqq \beta^{\prime} k\left(L_{0, H\left(x_{\theta}\right)}\left(u_{\theta}\right)\right)^{2}+(\delta / 2)\left(L_{m, H\left(x_{\theta}\right)}\left(u_{\theta}\right)\right)^{2},
$$

where $\beta^{\prime}$ is a constant independent of $g$. Combine (5.1) and (5.2) and use Lemma 1:

Now consider:

$$
\begin{aligned}
\left(L_{0, H\left(x_{\theta}\right)}\left(u_{g}\right)\right)^{2}-\left(L_{0, H\left(x_{\theta}\right)}\left(\widetilde{Q}\left(x_{g}, D, h\right) u_{g}\right)\right)^{2} & \\
& \geqq-\left|\beta^{\prime}\right| k\left(L_{0, H\left(x_{\theta}\right)}\left(u_{g}\right)\right)^{2}+(\delta / 2)\left(L_{m, H\left(x_{\theta}\right)}\left(u_{g}\right)\right)^{2} .
\end{aligned}
$$

$$
\begin{aligned}
& \left(L_{0, H\left(x_{\theta}\right)}\left(u_{g}\right)\right)^{2}-\left(L_{0, H\left(x_{\theta}\right)}\left(\widetilde{Q}(x, D, h) u_{g}\right)\right)^{2} \\
& \quad=\left(L_{0, H\left(x_{\theta}\right)}\left(u_{g}\right)\right)^{2}-\left(L_{0, H\left(x_{\theta}\right)}\left(\tilde{Q}\left(x_{g}, D, h\right) u_{g}\right)^{2}+\mathrm{IV}+\mathrm{V}+\mathrm{VI} .\right.
\end{aligned}
$$

Here

$$
\begin{aligned}
\mathrm{IV} & =2 \operatorname{Re}\left(\widetilde{Q}^{(0)} u_{g}, H\left(x_{g}\right)\left(\left(\widetilde{Q}\left(x_{g}, D, h\right)-\widetilde{Q}(x, D, h)\right) u_{g}\right)_{r},\right. \\
\mathrm{V} & =2 \operatorname{Re}\left(\left(\widetilde{Q}^{(1)}\left(x_{g}, D, h\right)+\widetilde{Q}^{(2)}\left(x_{\theta}, D, h\right)\right) u_{g}, H\left(x_{g}\right)\left(\widetilde{Q}\left(x_{g}, D, h\right)-\widetilde{Q}(x, D, h)\right) u_{g}\right)_{r}, \\
\mathrm{VI} & =-\left(L_{0, H\left(x_{\theta}\right)}\left(\left(\widetilde{Q}(x, D, h)-\widetilde{Q}\left(x_{g}, D, h\right)\right) u_{g}\right)\right)^{2} .
\end{aligned}
$$

Now choose $\epsilon$ in (4.2) sufficiently small. We can then estimate IV using Lemma 9, Schwarz's inequality, Lemmas 5, 6 and 8 obtaining:

$$
|\mathrm{IV}| \leqq \beta^{\prime \prime} k\left(L_{0, \boldsymbol{H}\left(x_{g}\right)}\left(u_{g}\right)\right)^{2}+(\delta / 12)\left(L_{m, H\left(x_{\theta}\right)}\left(u_{g}\right)\right)^{2} .
$$

It is easy to establish the same type of estimates for V and VI. Thus, using (5.3)

$$
\begin{aligned}
& \left(L_{0, H\left(x_{\theta}\right)}\left(u_{\theta}\right)\right)^{2}-\left(L_{0, H\left(x_{\theta}\right)}\left(\widetilde{Q}(x, D, h) u_{\vartheta}\right)\right)^{2} \\
& \geqq(\delta / 4)\left(L_{m, H\left(x_{g}\right)}\left(u_{g}\right)\right)^{2}+\beta^{\prime \prime \prime} k\left(L_{0, H\left(x_{\theta}\right)}\left(u_{g}\right)\right)^{2} .
\end{aligned}
$$


Finally consider:

$$
\begin{aligned}
& (u, H u)-(\widetilde{Q}(x, D, h) u, H \widetilde{Q}(x, D, h) u) \\
& =\sum_{g}\left(u_{g}, H\left(x_{g}\right) u_{o}\right)_{r}-\left((\tilde{Q}(x, D, h) u)_{o}, H\left(x_{g}\right)(\tilde{Q}(x, D, h) u)_{o}\right)_{r} \\
& =\sum_{o}\left(u_{o}, H\left(x_{o}\right) u_{o}\right)_{r}-\left(\widetilde{Q}(x, D, h) u_{g}, H\left(x_{o}\right) \tilde{Q}(x, D, h) u_{o}\right)_{r}-\text { VII }- \text { VIII } .
\end{aligned}
$$

Here

$$
\begin{aligned}
\mathrm{VII} & =2 \operatorname{Re} \sum_{g}\left(\widetilde{Q}(x, D, h) u_{g}, H\left(x_{g}\right)(\widetilde{Q}(x, D, h) u)_{o}-\widetilde{Q}(x, D, h) u_{g}\right)_{r}, \\
\mathrm{VIII} & =\sum_{g}\left(L_{0, H\left(x_{o}\right)}\left((\widetilde{Q}(x, D, h) u)_{o}-\widetilde{Q}(x, D, h) u_{g}\right)\right)^{2} .
\end{aligned}
$$

Because of the fact that all derivatives of $\phi(x)$ are uniformly bounded we easily show that,

$$
(\widetilde{Q}(x, D, h) u)_{o}-\widetilde{Q}(x, D, h) u_{o}=h \sum_{\tau, \nu} C_{\tau, \nu, o}(x) h^{\tau}(h D)^{\nu} u,
$$

where the elements of $C_{\tau, \nu, \theta}(x)$ have sufficiently many uniformly bounded derivatives. Observe that there exists a constant $N(h)$, decreasing with decreasing $h$, so that no more than $N(h)$ of the matrices $C_{\tau, \nu, o}(x)$ are different from 0 at any point $x \in R_{s}$. Using this, (5.4), and the same type of arguments as above we are now able to establish that

$$
(u, H u)-(\widetilde{Q}(x, D, h) u, H \widetilde{Q}(x, D, h) u) \geqq \beta^{\mathrm{Iv}} k(u, H u) .
$$

This proves Theorem 2 .

Royal Institute of Technology

Stockholm, Sweden

1. D. G. Aronson, "On the initial value problem for parabolic systems of differential equations," Bull. Amer. Math. Soc., v. 65, 1959, p. 310-318. MR 22 \#3888.

2. D. G. Aronson, "Parabolic equations with non-smooth coefficients," Duke Math. J., v. 29,1962 , p. $309-324$. MR $25 \# 5294$.

3. D. G. ARonson, "On the stability of certain finite difference approximations to parabolic systems of differential equations," Numer. Math., v. 5, 1963, p. 118-137; correction, ibid. v. 5,1963 , p. 290 . MR $27 \# 6408$.

4. S. D. EIDEL'MAN, "On fundamental solutions of parabolic systems," Mat. Sb. (N.S.), v. $38(80)$, 1956, p. 51-92. (Russian) MR 17, 857.

5. S. D. EIDEL'MAN, "On the fundamental solution of parabolic systems. II," Mat. Sb. (N.S.), v. 53 (95), 1961, p. 73-136. (Russian) MR 24 \#A925.

6. S. D. Eidel'man, "On Cauchy's problem for parabolic systems," Dokl. Akad. Nauk $S S S R$ v. 98, 1954, p. 913-915. (Russian) MR 17, 857.

7. L. GÅRDING, "Dirichlet's problem for linear elliptic partial differential equations," Math. Scand., v. 1, 1953, p. 53-72. MR 16, 366.

8. L. Hörmander, "On the uniqueness of the Cauchy problem," Math. Scand., v. 6, 1958, p. 213-225. MR $21 \# 3674$.

9. F. JoHN, "On integration of parabolic equations by difference methods. I," Comm. Pure Appl. Math., v. 5, 1952, p. 155-211. MR 13, 947.

10. H. O. KREISs, "Ưber die Lösung des Cauchyproblems für lineare partielle Differentialgleichungen mit Hilfe von Differenzengleichungen.I. Stabile Systeme von Differenzengleichungen," Acta Math., v. 101, 1959, p. 179-199. MR 24 \#A335.

11. H. O. KREISs, "Über die Differenzenapproximation hoher Genauigkeit bei Anfangsproblemen für partielle Differentialgleichungen," Numer. Math., v. 1, 1959, p. 186-202.

12. H. O. KREISS, "Ửber die Stabilitätsdefinition für Differenzengleichungen die partielle Differentialgleichungen approximieren," $B I T$, v. 2, 1962, p. 153-181. 
13. H. O. KRerss, "On difference approximations of the dissipative type for hyperbolic differential equations," Comm. Pure Appl. Math., v. 17, 1964, p. 335-353.

14. P. D. Lax \& B. Wendroff, "On the stability of difference schemes," Comm. Pure Appl. Math., v. 15, 1962, p. 363-371. MR 27 \#4375.

15. S. Mizohata, "Le probléme de Cauchy pour les équations paraboliques," J. Math. Soc. Japan, v. 8, 1956, p. 269-299. MR 19, 285.

16. S. Mizonata, Hypoellipticité des équations paraboliques," Bull. Soc. Math. France, v. 85,1957, p. $15-50$. MR $20 \# 3381$.

17. R. D. Richtm YeR, "Difference methods for initial-value problems," Interscience Tracts in Pure and Applied Mathematics, Tract 4, Interscience, New York, 1957. MR $20 \# 438$. 